\title{
Research on Application of Traditional Auspicious Elements in Modern Graphic Design
}

\section{Chenyuan Guo}

Yan' an University, Yan' an, Shaanxi, China, 716000

\author{
Keywords: Application, Traditional Auspicious Elements, Modern Graphic Design
}

\begin{abstract}
The traditional graphic art in China is extremely rich in resources. Starting with the basic features of traditional graphics, this paper will study how to excavate, change and transform it so as to better integrate the modern design concepts while maintaining its original traditional meaning. Graphic design has more profound connotation, more extensive artistic value, at the same time more vitality of the times. This paper analyzes and studies deeply the application of Chinese characters and graphics, traditional auspicious patterns and folk graphic in modern graphic design.
\end{abstract}

\section{Introduction}

The application of Chinese traditional auspicious text pattern in modern graphic design has become a common reality in our life. Such as the application of Chinese traditional auspicious patterns in modern furniture design; the application of traditional Chinese auspicious patterns in modern architecture design; the application of Chinese traditional auspicious patterns in the interior design of teahouse; the use of traditional Chinese auspicious text patterns in modern home textile designs Design applications and more. Its scope of application extends to all areas of life. The traditional Chinese auspicious writing pattern is an important part of the traditional Chinese culture and affects the aesthetic taste of the entire oriental nation. It has a special artistic value. It is a special graphic symbol closely linked to the needs and life of the Chinese people and represents people's aesthetic ideal and ideological pursuit. And combining such ideals and pursuits with the then production to create their own unique cultural system is an integral part of the Chinese nation's culture and a precious asset of the Chinese people with national characteristics. The combination of Chinese traditional auspicious text pattern and modern design makes contemporary design have its own national characteristics, and it is the protection, inheritance and development of Chinese traditional culture, which has the realistic significance of academic research.

\section{The Development Process and Its Connotation of the Auspicious Pattern}

During the Sui, Tang, Song and Yuan dynasties, the auspicious patterns in China were increasingly improved and gradually popularized. Especially in the Song and Yuan dynasties, Chinese auspicious patterns were widely used in architectural painting, ceramics, embroidery, fabrics and lacquer wares. The auspicious pattern of this time has entered a highly universal period of development and even reached the stage of "austerity" The point. Ming and Qing Dynasties, China auspicious patterns began to mature. The forms of auspicious patterns in China are more varied and colorful, and they are expressed in the form of pictorial techniques to make the auspicious patterns in China more mature and perfect. As the auspicious patterns, auspicious customs, the spread of auspicious words more far-reaching, but also gradually deepen the impact of social culture. In general, the history of the development of traditional Chinese auspicious patterns is rooted in primitive humanities. It started in Shang and Zhou dynasties and developed rapidly in the Song and Yuan Dynasties. It reached a peak in the Ming and Qing Dynasties.

Traditional auspicious patterns are unique to traditional Chinese motifs and can best embody the pattern manifestations of the Chinese national spirit, national feelings and national aesthetic values. They have profound cultural connotations. Whether in China or abroad, according to the development characteristics of their own nationalities, the traditional auspicious patterns formed on 
behalf of the local national culture have their own characteristics and distinctions. However, they always cannot be separated from the three major characteristics of the pattern, that is, decorative, Practicality, technology. Chinese traditional auspicious patterns originated in Shang and Zhou Dynasties, began in the Qin and Han dynasties. Chinese traditional auspicious patterns, the most important part of Chinese folk culture, reflect the longing for the Chinese nation to aspire to a better life in long-term social life. They translate the traditional auspicious belief in auspiciousness in natural phenomena or social phenomena into Visual patterns, I believe auspicious patterns can avoid disaster, to bring auspicious side. Chinese traditional auspicious motifs focus on the five major themes of "happiness, fortune, life, joy and wealth". In the eyes of Chinese people, the auspicious motif embodies the people's good wishes and the vision of a happy life and is the basis of Chinese folk culture. The auspicious rich connotation and the truth of good, good and beautiful in the traditional auspicious design are the symbols that people entrust with their beautiful yearning. The connotation of the auspicious pattern is an abstract concept. The auspicious pattern is to abstract and visualize abstract concepts. Bright Chinese people with rich imagination and associative force, the abstract concept of the first physical and physical contact with each other, and then this real thing landscaping, and combined with other mascot, the ultimate effect is Let people read the abstract concept in the picture. Its special cultural connotation in the graphic design through the use of new patterns of interpretation, without cumbersome text, ideographic more direct and accurate, in modern graphic design has been more widely used.

\section{The Features of Traditional Chinese Auspicious Text Patterns}

The formation of the concept of line modeling in Chinese graphic design art is due firstly to such objective factors as materials and tools, such as the tools used to depict graphics and the brush used in writing. In the Primitive Society, many of the activities that people were engaged in were related to their survival and reproduction. For the sake of these two relations of production, primitive humans often exercised witchcraft, such as offering sacrifices to Witch God. It is their rock carving pattern that allows us to see the true meaning of the original cultural psychology, and the form of this rock carved figure is exposed to the world. The concept of a line of people no doubt that changes in the thickness of the line, Qu straight rigid, length mutual aid, priority, such as Yunnan Cangyuan rock paintings graphics. Whether it is characters, animals, text is characterized by yin and yang mixed lines. Of course, these circular forms also include point and surface modeling elements. Another important reason for this is that unlike China, the concept of modeling in China is mainly based on the principle of realism. It is the pursuit of a specific artistic conception of surpassing appearance "The beauty, and this is precisely a major feature of Chinese design modeling. Chinese traditional decorative patterns have their own modeling features and aesthetic taste, the pursuit of order and the law of decorative beauty. Doomed Chinese traditional patterns pay more attention to the basis of line modeling graphics decorative beauty. Art begins with symbolism. Most scholars study the original intention of art. Its birth is not for art or itself art. However, the symbolic images of traditional Chinese decorative patterns (animal patterns, plant patterns and geometric patterns) contain strong connotation and art related factors. Hegel, in his discussion of "symbolic art," said: "The symbol, though not unlike the simple notion, cannot express meaning properly, but since it is a symbol, it cannot be completely consistent with meaning because, on the one hand, the content Meaning and signifying that their image is, of course, coordinated with one another over a certain trait; and on the other hand, the image of the symbol has some other properties that are completely unrelated to the general meaning of the symbolic. The concept until modern art has always been applicable. Today, mankind's thinking is enough to grasp the relationship between the meaning of the image and still use the emotional image of the symbol instead of the abstract meaning. Human mental activity was originally a symbolic activity. Primitive society, people through a variety of witchcraft left traces. Human beings have the ability to create symbols, and all cultures are the result of the symbolization of human life. Like the language symbols, image symbols are important carriers of human cultural exchanges. Chinese traditional auspicious text pattern uses a unique artistic symbol to convey the conception and connotation that 
has some connection with the graphic, which is inherited from the cultural conceptions of the past generations. The connection between it and the actual life of mankind some interest related. In the long-term, evolving process, these realities gradually lose their original meaning and transform into the notion of symbols we see today.

\section{The Application of Traditional Chinese Auspicious Patterns in Modern Graphic Design}

Studying the performance characteristics of traditional Chinese auspicious patterns in graphic design is actually a study of how to express tradition in a modern way. With the widespread use of traditional Chinese auspicious patterns in graphic design, auspicious patterns in China have become symbols that abstract the concept of conceptualization in graphic design. The new nationality graphic is a new concept, manifestation, and symbol element of the traditional auspicious pattern in the modern graphic design. The use of cutting, breaking up, dislocation, to create a defect-shaped traditional auspicious patterns, but also can be simplified, it can be complicated Chinese traditional auspicious patterns. The fierce collision between the old and the new forms has prompted the application of the traditional Chinese auspicious patterns in modern graphic design. There are quite a few examples of auspicious patterns used in modern graphic design in China, but the most representative of today is the symbol of the 2008 Beijing Olympic Games. From the inception of the Olympic Games to the official completion of the Olympic emblem, each part shows the characteristics of China and is unanimously praised by the whole world. The Olympic emblem consists of the colors of the Olympic rings. It looks like the "Chinese knot" of Chinese traditional folk handicrafts, and also looks like a human form of Taijiquan. "Chinese knot" symbolizes auspiciousness and happiness, as well as the spirit of unity and solidarity of every Chinese people. Taijiquan is the quintessence of China. The entire pattern is flowing, harmonious and vivid, full of sportsmanship, symbolizing the solidarity and cooperation among people in the world, exchange, development, work together to create the profound meaning of the new century. Of course, there are other examples, such as: Bank of China is the representative of Chinese financial business community, requiring the manifestation of Chinese characteristics. The symbol of Bank of China uses the Chinese ancient money and "Zhong" as the basic shape. The shape of the ancient money is a circular and shaped frame design with a square hole in the middle and a vertical line up and down to become the shape of "Zhong" To the round, based on the economy, gives the impression that is concise, stable, easy to identify, profound meaning, quite Chinese style. The reason why the Bank of China sign so impressed people is that I still owe it to people's understanding of the shape of the ancient money that symbolizes wealth. The symbol of China Telecom is the Chinese traditional pattern of palindromes and "Zhong" as the basis for the development and change of the three-dimensional space formed by the pattern, which symbolizes the communication network in all directions, a symbol of China Telecom from time to time smooth, ubiquitous, But also the image of the characteristics of China Telecom: science and technology, modern, transfer, speed, development, the decorative effect of this pattern is strong, and has the Chinese characteristics and the sense of the times, it can be said that the use of Chinese traditional culture very modern Feel the classic.

\section{Conclusions}

As a special "symbol element", Chinese traditional auspicious patterns are getting more and more convergence with modern graphic design. It expresses a certain meaning with scouring image, and uses people's symbol recognition, association and other thinking ability to convey specific information. With the help of the traditional auspicious patterns rich in meaning and meaning in the modern graphic design, through artistic re-creation, people express in the beautiful form the deep-seated desire for good things. Their aesthetic taste and habits permeate deeply and are rooted in people among ideologies, people like to understand and promote because of their inheritance. Since the charm of Chinese traditional auspicious patterns has attracted more people's attention and concern, its combination of formal beauty and intrinsic beauty has given modern graphic designers unlimited creative inspiration and creative sources. 


\section{References}

[1] Xue Shengyan, Li Ying. On the use of ceramic fragments in landscape design [J]. Ceramic Science and Art, 2007, (05).

[2] Weng Mei. On the Aesthetic Significance of Waste Ceramics and Its Application in Design of Ring Art [J]. Journal of Shandong University of Technology (Social Science Edition), 2009, (3): $109-111$.

[3] Gwen Heeny, Brickworks. A \& C Black (publisher), University of Pennsylvania Press, 2003.

[4] Zhang Min. Anthony Gaudi's Architectural Mosaic Art [J]. Decoration, 2007, (02).

[5] Zhang Yushan. Building pottery in the public environment art application [J]. Chinese Ceramic, 2008, (05): 68 - 70. 